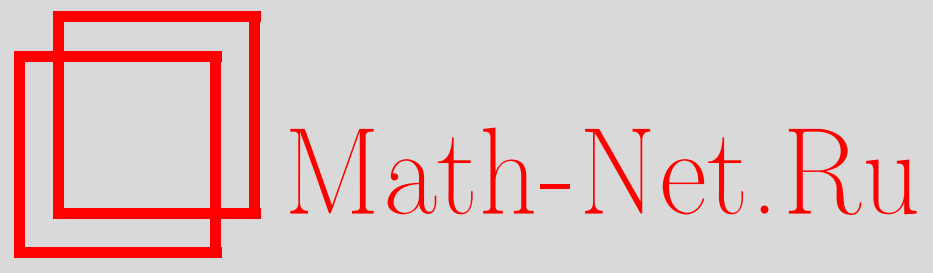

С. Н. Аристов, Е. Ю. Просвиряков, Об одном классе аналитических решений стационарной осесимметричной конвекции Бенара-Марангони вязкой несжимаемой жидкости, Вестн. Сам. гос. техн. ун-та. Сер. Физ.-мат. науки, 2013, выпуск 3()$, 110-118$

DOI: https://doi.org/10.14498/vsgtu1205

Использование Общероссийского математического портала MathNet.Ru подразумевает, что вы прочитали и согласны с пользовательским соглашением

http: //www.mathnet.ru/rus/agreement

Параметры загрузки:

IP : 35.173 .219 .12

26 апреля 2023 г., 16:11:04

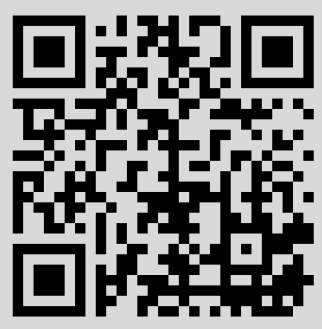




\title{
ОБ ОДНОМ КЛАССЕ АНАЛИТИЧЕСКИХ РЕШЕНИЙ СТАЦИОНАРНОЙ ОСЕСИММЕТРИЧНОЙ КОНВЕКЦИИ БЕНАРА-МАРАНГОНИ ВЯЗКОЙ НЕСЖИМАЕМОЙ ЖИДКОСТИ
}

\author{
C. Н. Аристов ${ }^{1}$, Е. Ю. Просвиряков ${ }^{2}$ \\ 1 Институт механики сплошных сред УрО РАН, \\ Россия, 614013, Пермь, ул. Акад. Королёва, 1. \\ 2 Казанский государственный национальный \\ исследовательский университет им. А. Н. Туполева, \\ Россия, 420111, Казань, ул. Карла Маркса, 10. \\ E-mails: asn@icmm.ru, evgen_pros@mail.ru
}

\begin{abstract}
Целъю настоящей работы является нахождение решений системы уравнений Обербека-Буссинеска плоской конвекиии Бенара-Марангони вязкой несжимаемой жидкости, в которых радиальная составляющая градиента температуры может обратитъся в нуль. Показано, что исходная система может бытъ сведена к системе обыкновенных дифберенциалъных уравнений одиннадиатого порядка. Получено точное решение в точке экстремума температуръ (при нулевом числе Грасгофа). Интегрирование уравнений осуществлено в безразмерных переменных, которые введены неклассическим способом: введен характерный масштаб по каждой переменной, а не по линейному характерному размеру слоя. Найденное решение служит начальным приближением для построения решения конвекции Бенара-Марангони при числах Грасгофа, больших, чем нуль.
\end{abstract}

Ключевые слова: осесимметричная термокапиллярная конвекиия (конвекиия Бенара-Марангони), локализованный параболический нагрев, точное решение, изолинии, матрица Гессе, собственнье числа, локализация корней полиномов, локализачия собственных чисел матрицы.

Введение. Не вызывает сомнений, что конвективное течение жидкости, связанное с неоднородным нагревом, является самым распространенным во Вселенной [1]. Систематические исследования конвективных течений вязкой несжимаемой жидкости датируются тысяча восемьсот восемьдесят восьмым годом, когда Томсон наблюдал образование сотовых структур в мыльной воде и мясном бульоне [2]. Это эмпирическое наблюдение легло в основу экспериментальной гидродинамики и регулярно используется для подтверждения или опровержения новых теоретических концепций, позволяющих количественно и качественно оценить основные характеристики течений.

В работах Бенара [3-5] отражено продолжение экспериментальных исследований конвективных течений и описана структура течения, представляющая собой пчелиные соты. При интерпретации опыта Бенаром в качестве механизма образования конвекции при подогревании плоского горизонтального слоя указывалась связь вязкости жидкости с термокапиллярными силами, возникающими на свободной поверхности [3-5], а не подъемная сила, как предполагали другие исследватели [1, 2]. Несмотря на то, что изучение конвекции жидкости началось с плоских течений, не меньший интерес вызывают осесимметричные течения. Осесимметричная конвекция БенараМарангони очень важна при изучении ряда технологических процессов, например, при описании выращивания кристаллов методом Чохральского [6], в

Сергей Николаевич Аристов (д.ф.-м.н.), главный научный сотрудник, лаб. гидродинамической устойчивости. Просвиряков Евгений Юръевич (к.ф.-м.н.), докторант, доцент, каф. основ конструирования. 
астрофизике [7], геофизической гидродинамике [7-10], метеорологии и климатологии $[7,9,10]$.

Точное решение уравнений, описывающих термокапиллярную конвекцию, впервые было найдено Бирихом [11]. Развитие и обобщение решения Бириха, интерпретацию физического смысла механизмов термокапиллярной конвекции можно проследить по работам [11-16]. Отметим, что до сих пор исчерпывающе не решена задача об описании осесимметричных термокапиллярных течений вблизи экстремальных значений температуры, в которых производная, вычисленная по радиальной координате, обращается в нуль. Построенные решения [11-16] не позволяют изучить анонсируемый класс течений, поэтому целью настоящей работы является построение точного решения системы Обербека-Буссинеска термокапиллярной конвекции, описывающего экстремальное температурное поле при числе Грасгофа, стремящемся к нулю. Найденное решение при необременительных ограничениях может быть использовано в качестве начального приближения для поиска устойчивых и неустойчивых решений осесимметричной конвекции Бенара-Марангони.

1. Постановка задачи. Запишем систему уравнений в цилиндрической системе координат, описывающих термокапиллярную конвекцию при установившемся осесимметричном движении вязкой несжимаемой жидкости (система уравнений Обербека-Буссинеска $[17,18])$ :

$$
\begin{aligned}
& V_{r} \frac{\partial V_{r}}{\partial r}+V_{z} \frac{\partial V_{z}}{\partial z}-\frac{V_{\varphi}^{2}}{r}=-\frac{\partial P}{\partial r}+\nu\left(\Delta V_{r}-\frac{V_{r}}{r^{2}}\right), \\
& V_{r} \frac{\partial V_{\varphi}}{\partial r}+V_{z} \frac{\partial V_{\varphi}}{\partial z}+\frac{V_{r} V_{\varphi}}{r}=\nu\left(\Delta V_{\varphi}-\frac{V_{\varphi}}{r^{2}}\right), \\
& V_{r} \frac{\partial V_{z}}{\partial r}+V_{z} \frac{\partial V_{z}}{\partial z}=-\frac{\partial P}{\partial z}+g \beta T+\nu \Delta V_{z}, \\
& V_{r} \frac{\partial T}{\partial r}+V_{z} \frac{\partial T}{\partial z}=\chi \Delta T, \quad \frac{1}{r} \frac{\partial\left(r V_{r}\right)}{\partial r}+\frac{\partial V_{z}}{\partial z}=0 .
\end{aligned}
$$

В системе уравнений (1) введены следующие обозначения: $V_{r}, V_{\varphi}, V_{z}$ - paдиальная, азимутальная и вертикальная скорости соответственно; $P$ - отклонение давления от гидростатического, делённое на постоянную среднюю плотность жидкости $\rho$; $T$-отклонение от средней температуры; $\nu, \chi-$ коэффициенты кинематической вязкости и температуропроводности жидкости соответственно; $\Delta=\frac{1}{r} \frac{\partial}{\partial r}\left(r \frac{\partial}{\partial r}\right)+\frac{\partial^{2}}{\partial z^{2}}-$ оператор Лапласа, определённый в цилиндрической системе координат.

При достаточно малом нагреве решения системы (1) могут быть разложены в ряды Тейлора с различным числом удерживаемых членов для анализа течений $[19,20]$. Решение системы (1) будем искать в следующем классе [19]:

$$
V_{r}=r u, \quad V_{\varphi}=r v, \quad V_{z}=w, \quad T=T_{0}+\frac{r^{2}}{2} T_{11}, \quad P=P_{0}+\frac{r^{2}}{2} P_{11} .
$$

Класс решений (2) обобщает решения, полученные Бирихом [11], Карманом [17], Сидоровым [20], Линем [21]. Подставим класс (2) в (1), получим полиномиальные выражения, которые в силу линейной независимости степенного базиса формируют систему уравнений, распадающуюся на две под- 
системы:

$$
\begin{gathered}
2 u+\frac{\partial w}{\partial z}=0, \quad \frac{\partial P_{11}}{\partial z}=g \beta T_{11}, \quad u^{2}+w \frac{\partial u}{\partial z}-v^{2}=-P_{11}+\nu \frac{\partial^{2} u}{\partial z^{2}} \\
2 u v+w \frac{\partial v}{\partial z}=\nu \frac{\partial^{2} v}{\partial z^{2}}, \\
2 u T_{11}+w \frac{\partial T_{11}}{\partial z}=\chi \frac{\partial^{2} T_{11}}{\partial z^{2}} \\
w \frac{\partial w}{\partial z}=-\frac{\partial P_{0}}{\partial z}+\nu \frac{\partial^{2} w}{\partial z^{2}}+g \beta T_{0}, \quad w \frac{\partial T_{0}}{\partial z}=\chi\left(T_{11}+\frac{\partial^{2} T_{0}}{\partial z^{2}}\right) .
\end{gathered}
$$

Запишем граничные условия, учитывая, что на верхней, свободной, границе задан локализованный источник тепла:

$$
T=\frac{\theta}{2}\left[1-\left(\frac{r}{R}\right)^{2}\right]
$$

Здесь $\theta$-характерная разность температур, $R$ - максимальное радиальное значение. Для основной системы (3) восьмого порядка граничные условия имеют следующий вид:

$$
\begin{aligned}
& \text { при } z=-h: v=w=\frac{\partial w}{\partial z}=0 ; \\
& \text { при } z=0: w=\frac{\partial v}{\partial z}=0, \quad P_{11}=0, \quad T_{11}=-\frac{\theta}{R^{2}}, \quad \frac{\partial u}{\partial z}=-\frac{\sigma}{\eta} T_{11},
\end{aligned}
$$

где $\sigma=\partial \alpha / \partial T$ температурный коэффициент поверхностного натяжения $\alpha$, $\eta$ - коэффициент динамической вязкости [18]. Для вспомогательной системы третьего порядка граничные условия имеют следующий вид:

$$
\text { при } z=-h: T_{0}+T_{11} \frac{R^{2}}{2}=0 ; \text { при } z=0: T_{0}=\frac{\theta}{2}, P_{0}=A \text {. }
$$

Здесь $A$ - атмосферное давление $[13,17,18]$. Граничное условие на твёрдой поверхности задается из условия, согласно которому имеет место нулевая температура на радиальной границе. Раскладывая в ряды Тейлора граничные условия, убеждаемся, что класс решений (2) удовлетворяет граничным условиям.

2. Решение редуцированной системы уравнений Обербека-Буссинеска. Для решения краевой задачи (3)-(6) приведем уравнения (3), (4) и граничные условия (5)-(6) к безразмерному виду. В качестве базиса выберем следующие размерные комплексы: $h$ - вертикальный (поперечный) характерный размер; $R$ - радиальный характерный размер; $\theta$ - температура; $g \beta \theta h^{4} /\left(\nu R^{2}\right)$ - вертикальная скорость; $g \beta \theta h$ - давление, делённое на постоянную плотность. Используя выбранный базис, получим краевую задачу, записанную в безразмерной форме для систем (3) и (4):

$$
\begin{array}{ll}
2 u+\frac{\partial w}{\partial z}=0, \quad \frac{\partial P_{11}}{\partial z}=T_{11} ; & \operatorname{Gr}\left(u^{2}+w \frac{\partial u}{\partial z}-v^{2}\right)=-P_{11}+\frac{\partial^{2} u}{\partial z^{2}} \\
\operatorname{Gr}\left(2 u v+w \frac{\partial v}{\partial z}\right)=\frac{\partial^{2} v}{\partial z^{2}}, & \operatorname{Gr}\left(2 u T_{11}+w \frac{\partial T_{11}}{\partial z}\right)=\frac{1}{\operatorname{Pr}} \frac{\partial^{2} T_{11}}{\partial z^{2}}
\end{array}
$$




$$
\begin{gathered}
\operatorname{Gr} \delta^{2} w \frac{\partial w}{\partial z}=-\frac{\partial P_{0}}{\partial z}+\delta^{2} \frac{\partial^{2} w}{\partial z^{2}}+T_{0}, \quad \operatorname{Gr} w \frac{\partial T_{0}}{\partial z}=\frac{1}{\operatorname{Pr}}\left(\delta^{2} T_{11}+\frac{\partial^{2} T_{0}}{\partial z^{2}}\right) ; \\
\text { при } z=-1: v=w=\frac{\partial w}{\partial z}=0 ; \\
\text { при } z=0: w=\frac{\partial v}{\partial z}=0, \quad P_{11}=0, \quad T_{11}=-1, \quad \frac{\partial u}{\partial z}=-\operatorname{Mg} T_{11} ; \\
\text { при } z=-1: T_{0}+\frac{T_{11}}{2}=0 ; \text { при } z=0: T_{0}=\frac{1}{2}, P_{0}=1 .
\end{gathered}
$$

Здесь $\mathrm{Gr}=g \beta \theta h^{5} /\left(\nu^{2} R^{2}\right)$ - осесимметричное число Грасгофа, записанное для вертикального и радиального характерных масштабов; $\operatorname{Pr}=\nu / \chi$ и $\mathrm{Mg}=$ $=\sigma /\left(\rho h^{2} g \beta\right)$ - число Прандтля и Марангони соответственно; $\delta=h / R$ - безразмерный геометрический параметр. Отметим, что если осуществлять обезразмеривание с одним характерным масштабом, то есть $h=R$, то получим классическое число Грасгофа: $\mathrm{Gr}=g \beta \theta h^{3} / \nu^{2}[1,2,6-18,20]$.

При числе Грасгофа, равном нулю $(\mathrm{Gr}=0)$, краевая задача $(7)-(9)$ имеет аналитическое решение:

$$
\begin{aligned}
& T_{11}=15\left(\mathrm{Mg}-\frac{1}{4}\right) z-1, \quad P_{11}=15\left(\mathrm{Mg}-\frac{1}{4}\right) \frac{z^{2}}{2}-z, \\
& u=15\left(\operatorname{Mg}-\frac{1}{4}\right) \frac{z^{4}}{4 !}-\frac{z^{3}}{3 !}+\operatorname{Mg} z+\frac{3}{8} \operatorname{Mg}-\frac{1}{96}, \\
& w=15\left(-2 \operatorname{Mg}+\frac{1}{2}\right) \frac{z^{5}}{5 !}+2 \frac{z^{4}}{4 !}-2 \operatorname{Mg} \frac{z^{2}}{2}+\left(-\frac{3}{4} \operatorname{Mg}+\frac{1}{48}\right), \\
& T_{0}=15 \delta^{2}\left(\frac{1}{4}-\mathrm{Mg}\right) \frac{z^{3}}{3 !}+\delta^{2} \frac{z^{2}}{2 !}+\left(\frac{15}{8}-\frac{15}{2} \mathrm{Mg}+\frac{5}{2} \operatorname{Mg} \delta^{2}-\frac{\delta^{2}}{8}\right) z+\frac{1}{2}, \\
& P_{0}=45 \delta^{2}\left(\frac{1}{4}-\mathrm{Mg}\right) \frac{z^{4}}{4 !}+3 \delta^{2} \frac{z^{3}}{3 !}+\left(\frac{15}{8}-\frac{15}{2} \operatorname{Mg}+\frac{5}{2} \operatorname{Mg} \delta^{2}-\frac{\delta^{2}}{8}\right) \frac{z^{2}}{2 !}+ \\
& +\left(\frac{1}{2}-2 \delta^{2} \mathrm{Mg}\right) z+1 \text {. }
\end{aligned}
$$

3. Исследование полученных решений. Проанализируем знаки значений гидродинамических полей (10). Рассмотрим для начала вертикальную скорость $V_{z}=w$. Найденное решение принадлежит классу многочленов пятой степени. Отметим, что в силу граничных условий (8), (9) многочлен $w$ можно факторизовать следующим образом [22]:

$$
w=z(z+1)^{2} g=z(z+1)^{2}\left[\left(\frac{1}{16}-\frac{\mathrm{Mg}}{4}\right) z^{2}+\left(\frac{\mathrm{Mg}}{2}-\frac{1}{24}\right) z+\frac{1}{48}-\frac{3 \mathrm{Mg}}{4}\right] .
$$

Поскольку координата $z$ определена на отрезке $[-1 ; 0]$, то $z(z+1)^{2} \leqslant 0$, следовательно, знак скорости будет определяться знаком квадратичного полинома $g$, коэффициенты которого непрерывно зависят от числа Марангони. $\Pi$ ри $\mathrm{Mg} \in[0 ; 1 / 36]$ скорость $w$ принимает только отрицательные значения на области определения координаты (рис. 1$)$, при $\mathrm{Mg} \in(1 / 36 ; 1 / 12)$ - как положительные, так и отрицательные значения, а при $\mathrm{Mg} \geqslant 1 / 12$ - положительные значения (рис. 1). Таким образом, в зависимости от числа Марангони на 
свободной (верхней) и твёрдой (нижней) границах скорость может одновременно иметь разные знаки (рис. 1). Полученный эффект смены знака вертикальной скорости на свободной границе при изменении числа Марангони наблюдался в экспериментальных и теоретических исследованиях $[1,11,13$, $18]$.

Знак радиальной скорости $V_{r}=r u$, очевидно, зависит от знака полинома $u$, который факторизуется следующим образом:

$$
\begin{aligned}
u=(z+1) s=(z+1)\left[\left(\frac{5 \mathrm{Mg}}{8}-\frac{5}{32}\right) z^{3}-\left(\frac{5 \mathrm{Mg}}{8}+\frac{1}{96}\right) z^{2}+\right. & \\
& \left.+\left(\frac{5 \mathrm{Mg}}{8}+\frac{1}{96}\right) z+\frac{3 \mathrm{Mg}}{8}-\frac{1}{96}\right] .
\end{aligned}
$$

На рис. 2 изображено множество значений функции $u$, что в силу класса (2) и области определения переменной $r \in[0 ; 1]$ с точностью до положительного множителя определяет множество значений радиальной скорости. При $\mathrm{Mg} \in[0 ; 1 / 36) \cup(1 / 12 ;+\infty)$ полином $s$ имеет один корень в области определения, а при $\mathrm{Mg} \in[1 / 36 ; 1 / 12]$ - два корня. Данная локализация корней поясняет геометрию множества значений радиальной скорости.

Используя решение (10), приведем (рис. 3) распределение температуры на твёрдой границе $(z=-1)$, зависящей, как нетрудно видеть, только от числа Марангони. Отметим, что на твёрдой границе при $\mathrm{Mg} \in[0 ; 11 / 60]$ жидкость имеет отрицательную температуру, при $\mathrm{Mg}>0$ - положительную. Если рассматривать $z \in(-1 ; 0)$, то знак и распределение температуры существенно зависят от величины $\delta$, причем существуют такие значения числа Марангони, что параметр $\delta$ принимает значения, большие шести. Это обстоятельство подчеркивает важность учета и введения различных масштабных параметров, а не одного [19].

Отметим, что для обобщённого давления можно провести анализ, аналогичный температурному, и уже на твёрдой границе $(z=-1)$ проявляется зависимость распределения знаков приведенного давления от числа Марангони $\mathrm{Mg}$ и параметра $\delta$.

На рис. 4-8 представлены изолинии обобщённой (модифицированной) функции тока [17], давления, отнесенного к плотности, и температуры при числе Марангони $\mathrm{Mg}=0$ и $\delta=1 / 2$.

Для уточнения типа изолиний, изображенных на рис. $4-8$, приведем алгоритм, позволяющий определить характер изолинии. Не ограничивая общности, рассмотрим алгоритм для температурного поля. Известно, что тип функции нескольких переменных (геометрически - поверхность) можно определить, анализируя знак гауссовой кривизны, который определяется знаками собственных значений ее матрицы Гессе [23-26]. Матрица Гессе, записанная для температуры, имеет следующий вид:

$$
H(T)=\left(\begin{array}{ll}
\frac{\partial^{2} T}{\partial r^{2}} & \frac{\partial^{2} T}{\partial r \partial z} \\
\frac{\partial^{2} T}{\partial z \partial r} & \frac{\partial^{2} T}{\partial z^{2}}
\end{array}\right)=\left(\begin{array}{cc}
T_{11} & r \frac{\partial T_{11}}{\partial z} \\
r \frac{\partial T_{11}}{\partial z} & \frac{\partial^{2} T_{0}}{\partial z^{2}}
\end{array}\right) .
$$

Изолинии температуры будут принадлежать к параболическому типу тогда, когда матрица Гессе имеет собственные значения одного знака, гиперболического - собственные числа разных знаков [23-26]. Воспользовавшись методами локализации собственных значений $[22,27,28]$, получим, что изолинии температуры и других гидродинамических полей могут принадлежать только к гиперболическому типу. 


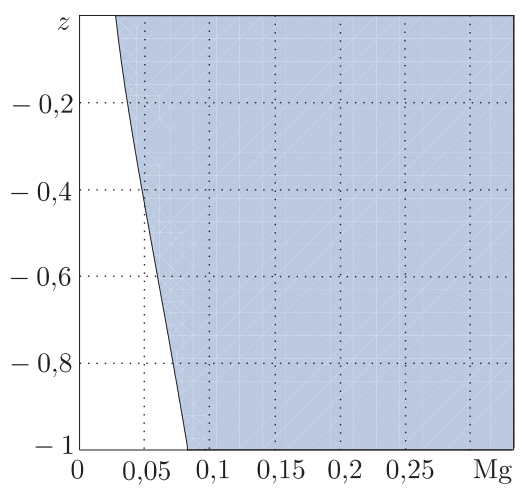

Рис. 1. Множество значений вертикальной скорости $w$ при различных числах Марангони Mg (закрашенная область соответствует положительным значениям, незакрашенная - отрицательным)

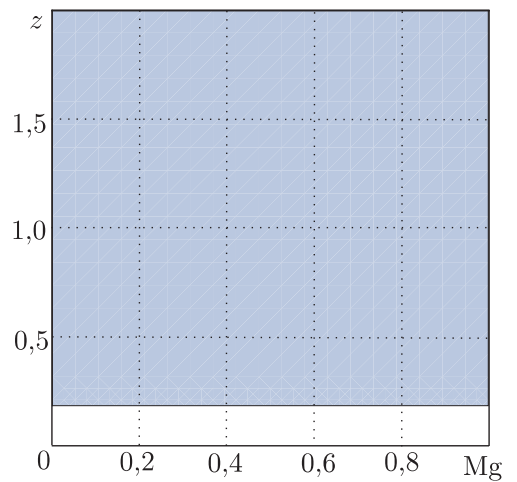

Рис. 3. Множество значений температуры на твёрдой границе при различных числах Марангони Mg (закрашенная область соответствует положительным значениям, незакрашенная - отрицательным)

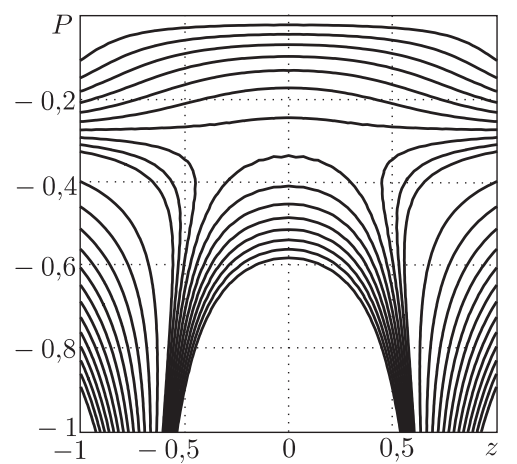

Рис. 5. Изолинии обобщённого давления при $\mathrm{Mg}=0,01, \delta=1 / 2$ (изолинии построены для значений, принадлежащих отрезку $[0,25 ; 0,9]$, с шагом, равным 0,05 ; для значений, принадлежащих отрезку $[0,9 ; 1]$ с шагом, равным 0,01$)$

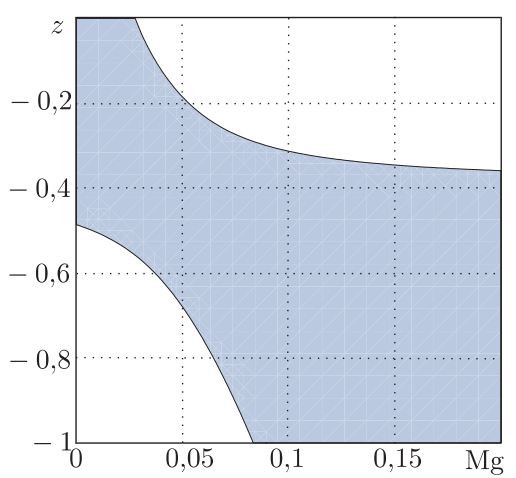

Рис. 2. Множество значений функции $u$ при различных числах Марангони $\mathrm{Mg}$ (закрашенная область соответствует положительным значениям, незакрашенная - отрицательным)

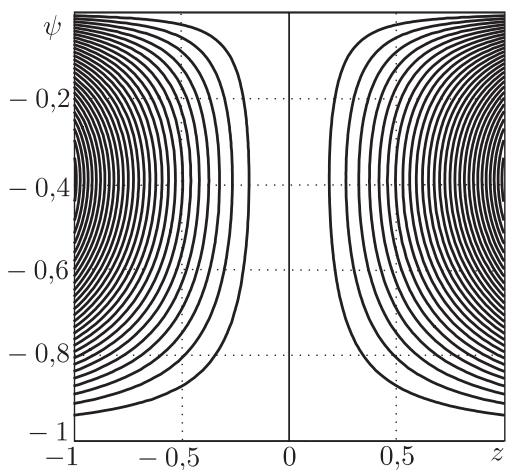

Рис. 4. Изолинии модифицированной функции тока при $\mathrm{Mg}=10, \delta=1 / 2$ (изолинии построены для значений, принадлежащих отрезку $[0 ; 1,4]$, с шагом, равным 0,1$)$

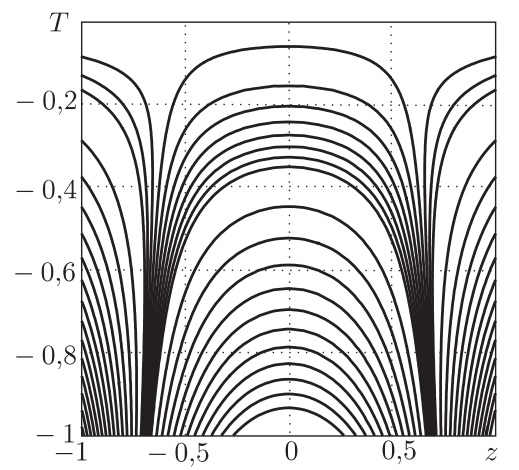

Рис. 6. Изолинии обобщённого давления при $\mathrm{Mg}=10, \delta=1 / 2$ (изолинии построены для значений, принадлежащих отрезку $[-30 ; 40]$, с шагом, равным 5) 


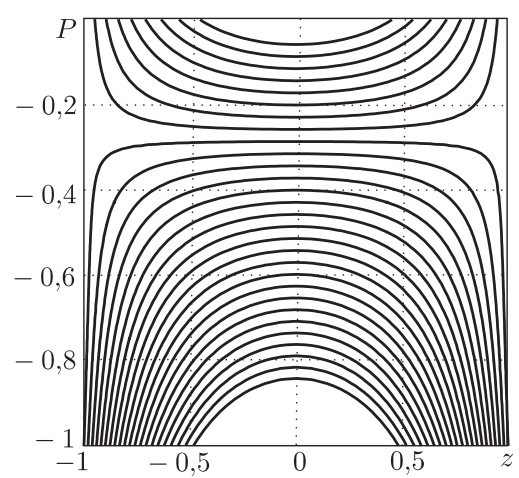

Рис. 7. Изолинии температуры при $\mathrm{Mg}=$ $=0,01, \delta=1 / 2$ (изолинии построены для значений, принадлежащих отрезку $[-1 ; 0,4]$, с шагом, равным 0,05$)$

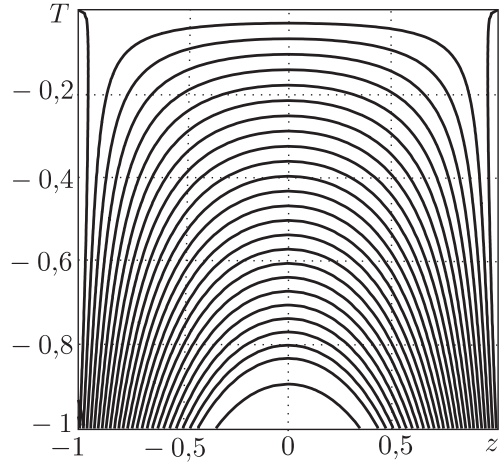

Рис. 8. Изолинии температуры при $\mathrm{Mg}=10, \delta=1 / 2$ (изолинии построены для значений, принадлежащих отрезку [0;60], с шагом, равным 5)

На рис. 5, 6 и рис. 7, 8 показана зависимость направления теплового потока от величины числа Марангони. Пограничное значение числа Марангони вычисляется методами локализации корней степенных решений (10), которые были использованы выше [22, 27, 28].

Заключение. Построено новое точное решение системы уравнений Обербека-Буссинеска, описывающее осесимметричное термокапиллярное течение вязкой несжимаемой жидкости при локальном параболическом нагреве свободной поверхности. Рассмотрен случай, когда число Грасгофа стремится к нулю. Определены области постоянств знаков у множеств значений решений в зависимости от числа Марангони и безразмерного параметра $\delta$. Найдено граничное значение числа Марангони, при котором происходит смена знака вертикальной и радиальной скоростей. Показано, что изолинии гидродинамических и температурного полей могут принадлежать только к гиперболическому типу.

Отметим, что полученные решения можно обобщить в следующих случаях. Во-первых, можно получить точные решения для расширенного класса (2), совершая преобразование сдвига для давления и температуры. Во-вторых, найденное решение может служить стартовым (начальным) решением при использовании итерационных методов нахождения точных решений конвекции Бенара-Релея при числах Грасгофа, больших нуля.

Работа выполнена при поддержке РФФИ (проект № 12-01-00023-а) и ФСР МФП НТС (программа СТАРТ).

\section{БИБЛИОГРАФИЧЕСКИЙ СПИСОК}

1. A. В. Гетлинг, "Формирование пространственных структур конвекции РэлеяБенара" // УФН, 1991. Т. 161, №9. С. 1-80; англ. пер.: A. V. Getling, "Formation of spatial structures in Rayleigh-Bénard convection"// Sov. Phys. Usp., 1991. Vol. 34, no. 9. Pp. 737-776.

2. Ф. А. Гарифуллин, "Возникновение конвекции в горизонтальных слоях жидкости" // Соросовск. образоват. ж., 2000. Т. 6, № 8. C. 108-114. [F. A. Garifullin, "Free convection in horizontal liquid layers" // Sorosovsk. Obrazovat. Zh., 2000. Vol.6, no. 8. Pp. 108-114].

3. H. Bénard, Les tourbillons cellulaires dans une nappe liquide propageant de la chaleur par convection, en régime permanent. Thèse. Paris: Gauthier-Villars, 1901. 88 pp.; Ann. de Chim. et Phys., 1901. Vol. 23. Pp. 62-144.

4. H. Bénard, "Étude expérimentale des courants de convection dans une nappe liquide. - 
Régime permanent: tourbillons cellulaires"// J. Phys. Theor. Appl., 1900. Vol.9, no. 1. Pp. 513-524.

5. H. Bénard, "Les tourbillons cellulaires dans une nappe liquide. - Méthodes optiques d'observation et d'enregistrement" // J. Phys. Theor. Appl., 1901. Vol. 10, no. 1. Pp. 254-266.

6. Н. В. Никитин, С. А. Никитин, В. И. Полежаев, "Конвективные неустойчивости в гидродинамической модели роста кристаллов методом Чохральского" // $y_{c n}$. мех., 2003. T. 2, № 4. C. 3-45. [N. V. Nikitin, S. A. Nikitin, V. I. Polezhaev, "Convective Instabilities in the Hydrodynamic Model of the Czochralski Crystal Growth" // Usp. Mekh., 2003. Vol. 2, no. 4. Pp. 3-45].

7. Г. С. Голицин, Природные процессы и явления: волны, планеты, конвекция, климат, статистика. М.: Физматлит, 2004. 344 с. [G. S. Golitsin, Natural processes and phenomena: waves, planets, convection, climate, statistics. Moscow: Fizmatlit, 2004. 344 pp.]

8. В. В. Алексеев, А. М. Гусев, "Свободная конвекция в геофизических процессах" // УФН, 1983. Т. 141, №2. С. 311-343; англ. пер.: V. V. Alekseev, A. M. Gusev, "Free convection in geophysical processes" // Sov. Phys. Usp., 1983. Vol.26, no. 2. Pp. 906-922.

9. А. С. Монин, Теоретические основы геофизической гидродинамики. Л.: Гидрометеоиздат, 1988. 424 с. [A. S. Monin, Fundamentals of Geophysical Fluid Dynamics. Leningrad: Gidrometeoizdat, 1988. 424 pp.]

10. А. Гилл, Динамика атмосферы и океана. Т. 1. М.: Мир, 1986. 397 с. [A. Gill, Atmosphereocean Dynamics. Vol. 1. Moscow: Mir, 1986. 397 pp.]

11. Р. В. Бирих, "О термокапиллярной конвекции в горизонтальном слое жидкости" // ПМТФ, 1966. № 3. С. 69-72; англ. пер.: R. V. Birikh, "Thermocapillary convection in a horizontal layer of liquid"// J. Appl. Mech. Tech. Phys., 1966. Vol. 7, no. 3. Pp. 43-44.

12. L. G. Napolitano, "Plane Marangoni-Poiseuille flow of two immissible fluids" // Acta Astronaut., 1980. Vol. 7, no. 4-5. Pp. 461-478.

13. В. К. Андреев, Решения Бириха уравнений конвекции и некоторые его обобщения: Препринт № 1-10. Красноярск: ИВМ СО РАН, 2010. 68 с. [V. K. Andreev, Birikh solution of convection equations and some of its generalizations: Preprint No. 1-10. Krasnoyarsk: Inst. Computer Modeling, Sib. Branch, Russian Acad. of Sci., 2010. 68 pp.]

14. O. Goncharova, O. Kabov, "Gas flow and thermocapillary effects of fluid flow dynamics in a horizontal layer" // Microgravity Sci. Technol., 2009. Vol. 21, no. 1. Pp. 129-137.

15. Л. Х. Ингель, М. В. Калашник, "Нетривиальные особенности гидротермодинамики морской воды и других стратифицированных растворов" // УФН, 2012. Т. 182, № 4. C. 379-406; англ. пер.: L. Kh. Ingel, M. V. Kalashnik, "Nontrivial features in the hydrodynamics of seawater and other stratified solutions" // Phys. Usp., 2012. Vol. 55, no. 4. Pp. 356-381.

16. В. С. Бескин, "Осесимметричные стационарные течения в астрофизике" // УФН, 2003. T. 173, №11. С. 1247-1253; англ. пер.: V. S. Beskin, "Axisymmetric steady flows in astrophysics" // Phys. Usp., 2003. Vol.46, no.11. Pp. 1209-1214.

17. Л. Д. Ландау, Е. М. Лифиии, Теоретическая физика. Т. VI: Гидродинамика. М.: Наука, 2006. 736 c. [L. D. Landau, E. M. Lifshits, Theoretical physics. Vol. VI: Fluid dynamics. Moscow: Nauka, 2006. 736 pp.]

18. Г. З. Гершуни, Е. М. Жуховицкий, Конвективная устойчивость несжимаемой жидкости. М.: Наука, 1972. 392 с. [G. Z. Gershuni, E. M. Zhukhovitskii, Convective stability of incompressible fluids. Moscow: Nauka, 1972. 392 pp.]

19. С. Н. Аристов, Вихревые течения в тонких слоях жидкости: Автореф. ... дис. докт. физ.-мат. наук.. Владивосток, 1990. 32 с. [S. N. Aristov, Eddy currents in thin liquid layers: Dr. Phys. \& Math. Sci. Thesis. Vladivostok, 1990. 32 pp.]

20. А. Ф. Сидоров, “Об одном классе решений уравнений газовой динамики и естественной конвекции" / В сб.: Численные и аналитические методы решения задач механики сплошной средъ; ред. А. Ф. Сидоров, Ю. Н. Кондюрин. Свердловск: УНЦ АН СССР, 1981. C. 101-117. [A. F. Sidorov, "On a class of solutions of the equations of gas dynamics and natural convection" / In: Numerical and analytical methods for solving problems of 
continuum mechanics; ed. A. F. Sidorov, Yu. N. Kondyurin. Sverdlovsk: Akad. Nauk SSSR, Ural. Nauchn. Tsentr, 1981. Pp. 101-117].

21. C. C. Lin, "Note on a class of exact solutions in magneto-hydrodynamics" // Arch. Rational Mech. Anal., 1958. Vol.1, no.1. Pp. 391-395.

22. E. E. Тыртышников, Матричный анализ и линейная алгебра. М.: Физматлит, 2007. 480 c. [E. E. Tyrtyshnikov, Matrix analysis and linear algebra. Moscow: Fizmatlit, 2007. 480 pp.]

23. С. П. Новиков, А. Т. Фоменко, Элементы дифференциальной геометрии и топологии. M.: Наука, 1987. 432 с. [S. P. Novikov, A. T. Fomenko, Elements of differential geometry and topology. Moscow: Nauka, 1987. 432 pp.]

24. В. И. Арнолъд, Теория катастроф. М.: Наука, 1990. 128 с. [V. I. Arnol'd, Catastrophe theory. Moscow: Nauka, 1990. 128 pp.]

25. Т. Постон, И. Стюарт, Теория катастроф и её приложения. М.: Мир, 1980. 608 с. [T. Poston, I. Stewart, Catastrophe theory and its applications. Moscow: Mir, 1980. 608 pp.]

26. Р. Гилмор, Прикладная теория катастроф. Т.1. М.: Мир, 1984. 350 с. [R. Gilmore, Catastrophe theory for scientists and engineers. Vol. 1. Moscow: Mir, 1984. 350 pp.]

27. Р. Хорн, Ч. Джонсон, Матричный анализ. М.: Мир, 1989. 655 c. [R. Khorn, Ch. Dzhonson, Matrix analysis. Moscow: Mir, 1989. 655 pp.]

28. M. М. Постников, Устойчивые многочлены. М.: Наука, 1981. 176 с. [M. M. Postnikov, Stable polynomials. Moscow: Nauka, 1981. 176 pp.]

Поступила в редакцию $22 / \mathrm{I} / 2013$;

в окончательном варианте - 21/III/2013.

MSC: 76F02, 76M45; 76F45, 76R05, 76U05

\section{ON ONE CLASS OF ANALYTIC SOLUTIONS OF THE STATIONARY AXISYMMETRIC CONVECTION BÉNARD-MARAGONI VISCOUS INCOMPREEIBLE FLUID}

\section{S. N. Aristov, E. Yu. Prosviryakov}

1 Institute of Continuous Media Mechanics UB RAS,

1, Academician Koroleva st., Perm, 614013, Russia.

2 Kazan National Research Technical University named after A. N. Tupolev,

10, Karl Marx st., Kazan, 420111, Russia.

E-mails: asn@icmm.ru, evgen_pros@mail.ru

The purpose of this work is to find solutions for the system of equations OberbeckBoussinesq flat convection Bénard-Marangoni a viscous incompressible fluid. In this viscous incompressible fluid the radial component of the temperature gradient may become zero. It is shown that the initial system may be reduced to the system of equations of ordinary differential equations of the eleventh order. We obtain the exact solution at the point of the extremum of the temperature (at zero including Grasgof's). Integration of equations is carried out in dimensionless variables, which are non-classical way: put the scale factor for each variable, and not by linear characteristic size of the layer. The solution is the initial approximation to the solution of convection Bénard-Marangoni in numbers Grasgof's, the big zero.

Key words: axisymmetric thermocapillary convection (convection Bénard-Marangoni), localized parabolic heaters, exact solution, isolines, Hessian matrix, eigenvalues, localization of polynomials roots, localization of eigenvalues of the matrix.

Original article submitted 22/I/2013; revision submitted $21 / \mathrm{III} / 2013$.

Sergey N. Aristov (Dr. Phys. \& Math. Sci.), Chief Researcher, Lab. Hydrodynamical Instability. Evgeii Yu. Prosviryakov (Ph. D. Phys. \& Math.), Doctoral Candidate, Dept. of Bases of Construction. 